\section{Control inteligente de un sistema de riego mediante asistente de voz}

\author{
Irrigation System Smart Control throughout voice \\ assistance
}

\section{Erika Katherine Gómez Llenera* \\ Davis Jefferson Sevilla Sanango* \\ Marcos Giovanny Orellana Parra* \\ Ana Gabriela Hinojosa Caballero*}

\section{RESUMEN}

El trabajo tuvo como objetivo diseñar un sistema de riego por aspersión, mediante asistente de voz para cambio de estado ON/OFF. Bajo las características de un proyecto factible, se orientó la investigación para resolver un problema planteado y a satisfacer las necesidades de una comunidad específica en cuanto al mantenimiento de sus áreas verdes. Se concluye, que el sistema diseñado es factible de implementar, bajo ciertas condiciones de conectividad con internet, lo cual se recomienda contar con un mando manual, en caso de deficiencias en la conexión.

Palabras clave: Control inteligente, sistema de riego por aspersión, asistente de voz

\section{ABSTRACT}

The objective of the work was to design a sprinkler irrigation system, using a voice assistant to change the $\mathrm{ON}$ / OFF state. Under the characteristics of a feasible project, the research was oriented to solve a problem posed and to satisfy the needs of a specific community regarding the maintenance of its green areas. It is concluded that the designed system is feasible to implement, under certain conditions of connectivity with the internet, which is

\footnotetext{
* Estudiante ingeniería TI, Universidad Católica de Cuenca, extensión La Troncal erika.gomez@est.ucacue.edu.ec, https://orcid.org/0000-0003-3/37-6652

* Estudiante ingeniería TI, Universidad Católica de Cuenca, extensión La Troncal davis.sevilla@est.ucacue.edu.ec, https://orcid.org/0000-0002-3896-0619

* Ingeniero en electrónica y telecomunicaciones, Universidad Católica de Cuenca, extensión

La Troncal, morellanap@ucacue.edu.ec, https://orcid.org/0000-0003-2976-3I6X

* ingeniera electrica, Universidad Católica de Cuenca, extensión La Troncal

aghinojosac@ucacue.edu.ec, https://orcid.org/0000-000I-6885-0384
}

\section{REVISTATECNOLÓGICA ciencia y educación Edwards Deming}

ISSN: 2600-5867

\section{Atribución/Reconocimiento-NoCo} mercial- Compartirlgual 4.0 Licencia Pública Internacional — CC

\section{BY-NC-SA 4.0}

https://creativecommons.org/licenses /by-nc- sa/4.0/legalcode.es

Editado por: Tecnológico Superior Corporativo Edwards Deming

Enero - Marzo Vol. 6 - I - 2022

https://revista-edwardsdeming.com/index.php/es e-ISSN: 2576-097I

Recibido: 27 junio 2021

Aprobado: 09 septiembre, 202।

Pag I - 12 
recommended to have a manual control, in case of deficiencies in the connection.

Keyword: Smart control, sprinkler irrigation system, voice assistant

\section{INTRODUCCIÓN}

A continuación, se presenta un proyecto el cual tuvo como objetivo general el control inteligente de un sistema de riego mediante asistente de voz, desarrollando para ello contenidos que permitieron identificar los sistemas de riego y los de asistencia de voz que permiten controlar dispositivos SMART, explicar el funcionamiento electromecánico de los relés; para luego diseñar la etapa de control para la operación del sistema de riego y su implementación.

Se espera que este sistema automatizado empleando WIFI relé ICH, Tuya Alexa, sea una alternativa útil para el riego por aspersión de aquellas unidades familiares e institucionales que requieran, desde una red inalámbrica dotar de mantenimiento adecuado a sus áreas verdes, bien sea ornamentales o de diversos cultivos.

\section{Sistemas de riego en el área de la agronomía.}

Según Pineda (s/f) un perímetro de riego, es el conjunto de sistemas estructurales que tienen como objetivo la aplicación del agua para que una zona determinada pueda ser cultivada y lograr el mantenimiento de las plantas.

En ese sentido, para Antúnez y col (2010), un sistema de riego, es la capacidad que se tiene por medio de emisores, en satisfacer la necesidad hídrica de las plantas, utilizando los factores agroclimáticos, herramientas, tecnología y materiales para su implementación, minimizando los desperdicios e impactos ambientales. Entre sus características se encuentran que a través de él se ahorra agua dependiendo del tipo de sistema, hay un control adecuado, reduce problemas de obturación, se logra una resistencia a la tracción, el riego es uniforme, tanto en superficie o enterrado, ahorro de energía, y bajo costo de mano de obra por la automatización.

De acuerdo a Demin (20l4), el sistema de riego está compuesto principalmente por dos tipos: por inundación y surcos (no presurizado) y sub-foliar (presurizados). En el primero el agua es aplicada mediante pequeños surcos entre dos líneas de siembra; el agua se infiltra y llega alrededor de la zona de las raíces del cultivo. El segundo, permite que el agua llegue a las plantas o a los cultivos que se pretenden regar, de una manera localizada, donde recibe la cantidad suficiente sin que haya excesos o déficit de ésta. A este grupo pertenecen los siguientes sistemas de riego: por aspersión, por nebulizadores, por pívot y por goteo.

Para efectos de este trabajo, se considera el riego sub-foliar (presurizados), en su modalidad por aspersión, el cual consiste en incorporar agua a alta presión, en forma de lluvia sobre las plantas. Con el empleo de energía eléctrica, el agua es conducida por una manguera a fin de cubrir toda el área sembrada y al llegar al aspersor, el caudal se rompe en muchas gotas que caen sobre el suelo. 
Figura I. Sistema de riego por aspersión

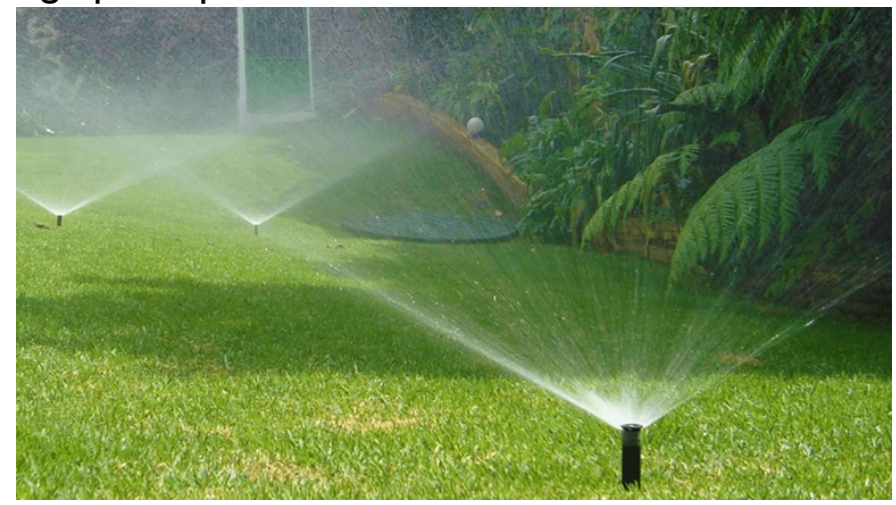

Fuente: Elaboración propia.

Tomado de:

https://www.google.com/search?q=riego+por+aspersi\%C3\%B3n\&sxsrf=ALeKk03HleN w

\section{Sistemas de asistencia de voz que permiten controlar dispositivos SMART.}

Para Pelaez (s/f), la tecnología aplicada a la agricultura, se conoce también con el término de agrotecnología, y ha tenido gran cabida en los últimos tiempos debido a las aplicaciones que se han desarrollado para llevar a cabo las prácticas agrícolas. Estas aplicaciones han dado múltiples ventajas a los productores de tierras, razón por la cual con la llegada de las nuevas tecnologías a este sector se disminuyó en gran parte las cargas de trabajo pesado para los agricultores y estos aumentaron su producción.

Peláez (s/f), señala que hay diversas nuevas tecnologías dentro del sector agrícola, como sembradoras y tractores con GPS, agricultura de alta precisión, nuevas tecnologías aplicadas a la recolección, Drones, sistemas de riego telemático. Es precisamente este último el de interés para este trabajo de investigación, donde se observa una de las ventajas en su implementación, ya que, mediante la introducción de sistemas telemáticos, los agricultores pueden controlar el riego de sus plantas desde cualquier lugar, ahorrando tanto dinero como tiempos de desplazamiento.

En ese sentido, la comunicación inalámbrica, está siendo empleada como enlace entre distintos dispositivos como bluetooth, infrarrojos, wifi, entre otros; permitiendo así a través de la tecnificación ser más productivos. También se cuenta con los sistemas de asistencia de voz que permiten controlar dispositivos SMART, que pueden ser utilizados en el sistema de riego. 
Para Arganza y Arroyo (2019), los asistentes de voz son programas de software basados en la inteligencia artificial que son capaces de reconocer el lenguaje con una elevada precisión (gracias al machine learning) y responder a comandos de voz para ejecutar una serie de tareas y preguntas según las fuentes de información disponibles, de modo que posibilitan a los usuarios interactuar con diferentes plataformas y hardware mediante la voz. Estos pueden estar integrados en diversos tipos de plataformas y dispositivos, entre ellos: Altavoces inteligentes, smartphones o teléfonos inteligentes, smartwatch y wearables, google assistant, entre otros.

Existen diferentes asistentes de voz que permiten controlar dispositivos SMART, entre ellos se encuentran: Asistente de Google (Google Assistant); Alexa, un asistente virtual desarrollado por Amazon; Cortana, asistente virtual de Microsoft, y Siri, sistema de reconocimiento de voz de Apple.

En este sentido, se debe precisar que, para la investigación, se empleó el asistente de voz Alexa, a través del cual se puede controlar varios dispositivos inteligentes que sean compatibles con este sistema, ejemplo de ello se encuentran focos, cámaras de video vigilancia, interruptores inteligentes, relés electromecánicos entre otros. $Y$ donde los usuarios pueden extender las habilidades de Alexa instalando "skills" (funcionalidades adicionales desarrolladas por terceros parecidas a las aplicaciones) desde la app Alexa, y crear rutinas para automatizar sus dispositivos inteligentes con base a un comando de voz, hora o ubicación y tiempo.

\section{Funcionamiento electromecánico de los relés.}

De acuerdo a Cinjordiz (2016), el principal funcionamiento de un relé es el de activar, poner en marcha o encender algo mediante una señal eléctrica con una intensidad mucho menor que la intensidad que va a consumir el aparato o receptor que queremos encender o poner en marcha. Una característica importante de estos dispositivos, es que la parte que emite la señal para activar el relé está aislada de la parte que pone en marcha o enciende el receptor. Existen varios tipos de relés dependiendo del uso que se le desee dar, entre ellos: los polarizados, multitensión, relés electromagnéticos, entre otros.

En este proyecto se empleó WIFI relé $\mathrm{ICH}$, Tuya Alexa, la cual mediante ordenes previamente configuradas acciona el sistema de riego en un espacio determinado. El dispositivo empleado es un módulo relé de un pulso, controlado desde el celular y por comandos de voz con Alexa.

\section{MATERIALES Y MÉTODOS}

Este trabajo está desarrollado bajo el tipo de investigación denominado proyecto factible; el cual según Balestrini (2002, p. 9), son aquellos "proyectos o investigaciones que proponen la formulación de modelos, sistemas entre otros, que dan soluciones a una realidad o problemática real planteada, la cual fue sometida con anterioridad o 
estudios de las necesidades a satisfacer". Por otra parte, se está en presencia de un estudio de carácter experimental, el cual de acuerdo a Hernández y col. (20/4), la esencia de esta concepción de experimento es la manipulación intencional de una acción para analizar sus posibles resultados.

En el mismo orden de ideas, para diseñar e implementar el sistema de riego por aspersión, mediante asistente de voz para cambio de estado ON/OFF, se hizo necesario acudir, en primera instancia a fuentes secundarias, a través de la revisión de libros de agronomía, artículos, folletos, entre otros recursos documentales que permitieron conocer con más precisión, los diferentes sistemas de riego que se emplean y los sistemas y dispositivos electromecánicos que se pudieran utilizar. Para luego, diseñar todo el sistema y hacer el trabajo de campo o el experimento requerido, para constatar su funcionamiento.

De esta manera, se diseñó el sistema de riego por aspersión mediante asistente de voz empleando WIFI relé ICH, Tuya Alexa, y el funcionamiento del mismo fue probado en las áreas verdes que circundan a la Universidad Católica de Cuenca extensión San Pablo de a Troncal.

\section{RESULTADOS}

\section{Diseño de control para la operación del sistema de riego.}

El sistema de riego se implementa con la ayuda de un dispositivo WIFI Smart ICH Relé, propietario de TUYA Smart Home, en las ilustraciones que se muestran de inmediato, se pueden observar las características físicas y técnicas:

Figura 2. Especificaciones físicas

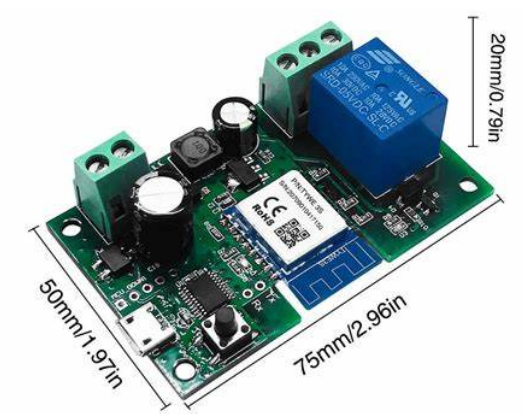

Fuente: manual de usuario WiFi Smart ICH Relay

Lista de verificación antes de usar el dispositivo:

$\checkmark \quad$ El adaptador de corriente USB debería ser máximo de $500 \mathrm{~mA}$ de salida

$\checkmark \quad$ El teléfono inteligente o tableta debe tener conectado un WIFI 2.4G con internet 
$\checkmark \quad$ El dispositivo solo admite Wi-Fi 2.4G. Si utiliza un enrutador 5G (proporciona dos señales WI-Fl: $5 \mathrm{G}$ y $2.4 \mathrm{G}$ ), seleccione el $2.4 \mathrm{G}$ WIFi para conectar su teléfono inteligente

$\checkmark \quad$ Descarga e instala la aplicación Tuya Smart o la aplicación Smart Life de la tienda de aplicaciones o Tienda Google Play. Regístrese e inicie sesión

$\checkmark \quad$ Durante el proceso de emparejamiento, asegúrese de que el dispositivo IOS o Android y el dispositivo este dentro del alcance de su enrutador WIFi.

$\checkmark \quad$ Asegúrese de que su enrutador sea MAC Open. De lo contrario, cancele primero la configuración de filtrado MAC del enrutador

Figura 3. Especificaciones técnicas

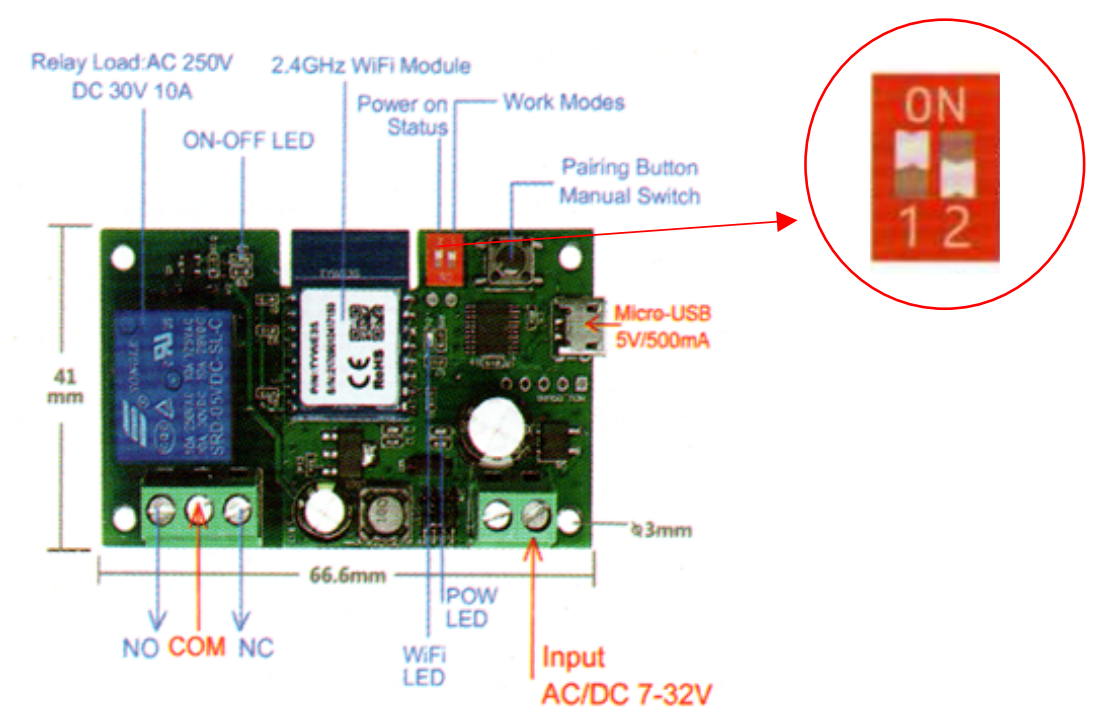

Fuente: manual de usuario WiFi Smart ICH Relay

$>$ Función de interruptor de modos de trabajo y configuración de estado de encendido

$\mathrm{I}=\mathrm{ON}=$ Inching

$I \neq O N=$ Selflock

$2=\mathrm{ON}=$ Power up $=\mathrm{ON}$

$2 \neq$ ON $=$ Power up $=$ OFF

Inching: el relé se activa durante $0,7 \mathrm{~s}$

Selflock: el relé se activa normalmente ENCENDIDO / APAGADO

Estado de encendido: una vez que el dispositivo está encendido, el relé puede ser activado o no. 
> Modo de autobloqueo:

Una vez que se pulsa el interruptor manual o control desde la aplicación, el relé se activa para mantener encendido o apagado normalmente

Se puede usar para controlar la luz / ventilador/ humidificador u otros electrodomésticos.

$>\quad$ Modo de avance lento: Inching mode

Una vez que presione el interruptor manual o control desde la aplicación, mantenga presionado durante 7 segundos y luego suéltelo automáticamente.

La versión WiFi + RF admite configurar tiempo de avance de Is-Ih en el temporizador de la aplicación (Pero configure el dispositivo en modo de autobloqueo)

Se puede utilizar para controlar puertas / abrepuertas / ascensor / ordenador / tecla del mando a distancia / cualquier botón de retardo. (Extraído del manual del Tuya Smart).

$>\quad$ Otros equipos necesarios

Bomba de agua: Se dispone para la operación de una bomba de agua de marca Pedrollo de $1 / 2 \mathrm{HP}$ - IIO V, un aspersor de $1 / 2$ ", elementos de conexión manguera y conductores eléctricos.

Figura I. Bomba de agua I/2 HP-I IOV

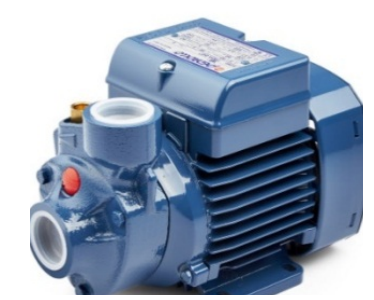

Fuente: Elaboración propia. Tomado de https://www.google.com/search?q=Bomb a+de+agua

Figura 5. Conductor Flex I2AWG

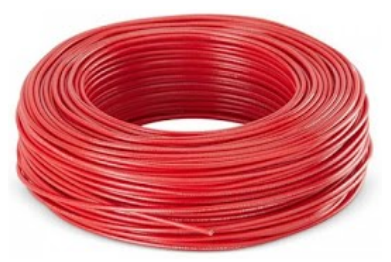

Fuente: Elaboración propia. Tomado de https://www.google.com/search?q=Cond uctor+Flex+ I 2AWG\&tbm
Figura 4. Aspersor de agua I/2"

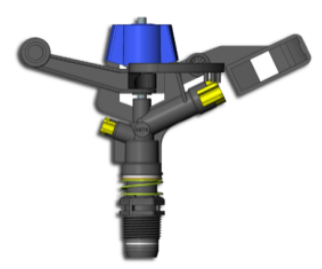

Fuente: Elaboración propia. Tomado de https://www.google.com/search?q=Asper sor+de+agua+ I/2"\&hl

Figura 6. Manguera de agua I/2”

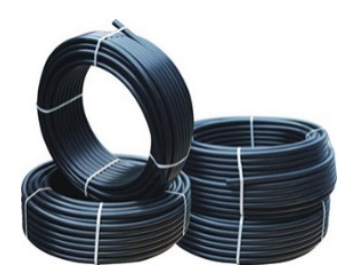

Fuente: Elaboración propia. Tomado de https://www.google.com/search?q=mang uera + agua + media+pulgada 
Para la conexión se utiliza el modulo Smart Tuya alimentado con I2vdc a través de un transformador de mando y control; el circuito posee un selector de dos posiciones para poner en marcha de forma manual. Se cuenta además, con un pulsador de paro de emergencia el que estará colocado cerca del tablero de control, y una protección térmica para el motor eléctrico.

Figura 7. Diagrama de control para bomba de agua utilizando módulo de control wi-fi

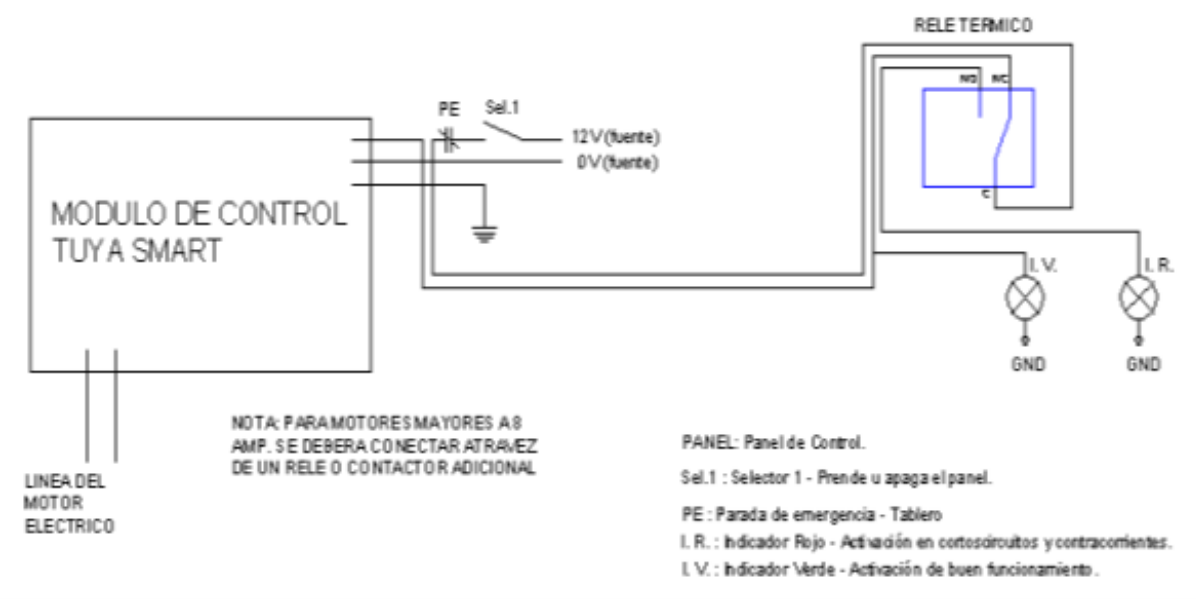

Fuente: Elaboración propia

En la configuración de control se requiere un router con wi-fi, además de un dispositivo Smartphone (Tablet, teléfono, etc.). Estos se conectan inalámbricamente, y pueden controlarse desde cualquier lugar con un dispositivo con acceso a internet.

Figura 8. Mando desde móvil Smartphone para el control con Tuya Smart y Alexa de Amazon

Fuente: Elaboración propia 
Para el diagrama de fuerza se emplean interruptores o breakers para la protección general, y para cada circuito, control y fuerza, los calibres de conductores utilizados para esta conexión son el diagrama de fuerza e interruptores o breakers para la protección general.

Para cada circuito, control y fuerza, los calibres de conductores utilizados para esta conexión son \# 12 awg; sin embargo, dependiendo de la potencia del motor se deberá diseñara la capacidad de los breakers, relé térmico y conductores.

Figura 9. Diagrama de Fuerza la red I20v AC.

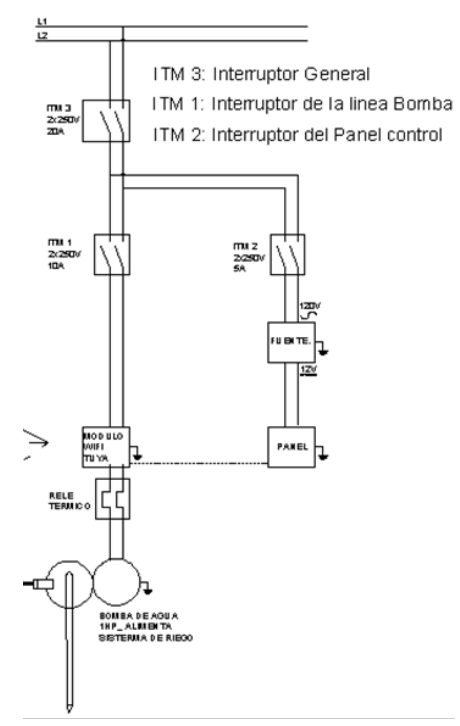

Fuente: Elaboración propia

El motor eléctrico acciona mecánicamente a una bomba de agua de presión, esta a su vez empujara el agua a través de las tuberías, hasta los aspersores que se encargan de regar el agua a las plantaciones.

Figura 10. Diagrama de instalación de aspersores

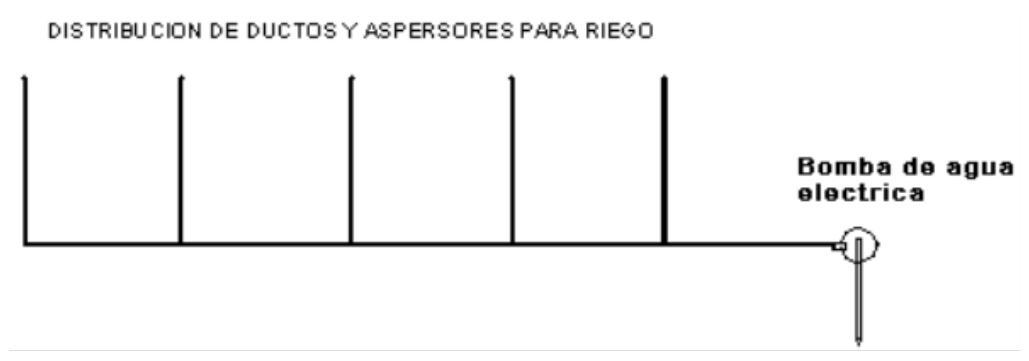

Fuente: Elaboración propia 
Para el funcionamiento y control del sistema de riego con aspersores, se considera la disponibilidad del agua, por lo que se deberán adicionar sensores de nivel de agua, en caso de tomar de una cisterna o pozo; además válvulas en cada derivación a los aspersores por mantenimiento y una válvula cheque antes de la bomba para evitar la descarga de agua del sistema.

\section{Implementación de control para la operación del sistema de riego.}

Para la implementación del sistema de riego automatizado por control de voz, se utilizaron los dispositivos, equipos y materiales señalados en párrafos anteriores. Los mimos fueron dispuestos en un espacio abierto, dentro de las instalaciones de la Universidad Católica de Cuenca extensión San Pablo de a Troncal. En detalle se indica que la bomba de agua de $1 / 2 \mathrm{HP}$ - $110 \mathrm{~V}$, se conecta a un pozo profundo, por ser una versión preliminar o de prueba, no se incluye una válvula cheque para evitar la descarga de agua del sistema, además del filtrado del agua que ingresa a la bomba.

Se considera un solo breaker para protección de todo el circuito, no se tomó en cuenta el guarda motor, sin embargo cada uno de estos accesorios serán considerados en el montaje definitivo del proyecto a fin de brindar protección al sistema. Por otra parte, mediante la aplicación Tuya Smart, se establece la conexión WIFI a la red de la Universidad, que opera a $2.4 \mathrm{GHZ}$, y se verifica el reconocimiento de los dispositivos para la adecuada ejecución del sistema. Una vez establecido los dispositivos en la Tuya Smart y conectado todo el sistema de riego por aspersión se realizaron las pruebas necesarias para verificar el funcionamiento correcto del sistema de riego. 
Figura I I. Diagrama de conexión de sistema de riego por aspersión automatizado

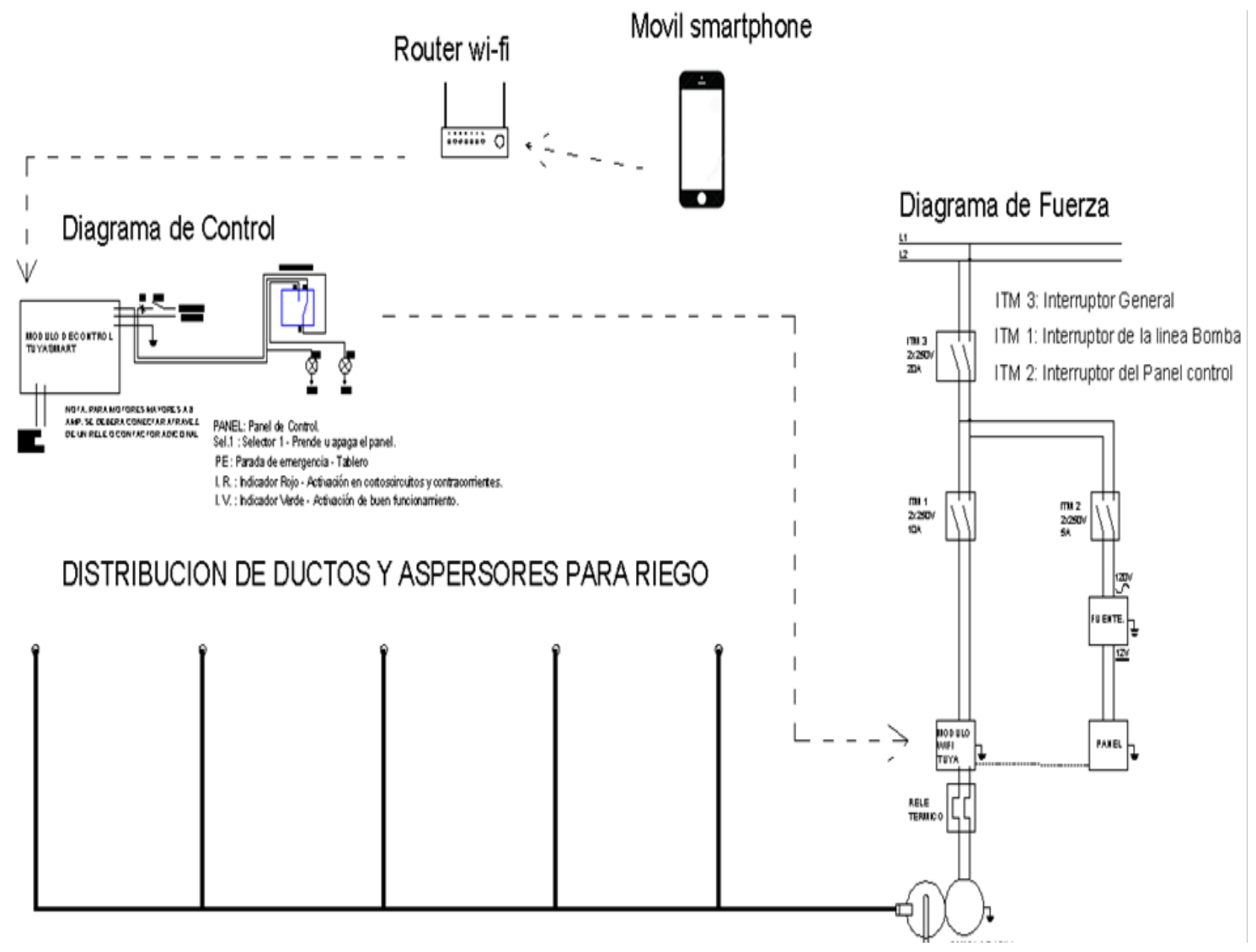

Fuente: Elaboración propia

\section{DISCUSIÓN}

En este proyecto se ha diseñado, implementado y comprobando un sistema automatizado empleando WIFI relé ICH, Tuya Alexa, el cual mediante órdenes previamente configuradas acciona un sistema de riego empleando mandos de voz, con la ayuda de redes inalámbricas y conectividad a Internet se evita el cableado de datos hasta el lugar donde se realiza la acción de regado. El mismo se vislumbra con fuertes ventajas y oportunidades de ser implementado en los hogares y organizaciones que cuentan con un espacio destinado a las áreas verdes.

Una limitante del proyecto es que debe ser implementado en una zona con acceso o servicio de Internet, no se podrá controlar el dispositivo de manera eficiente si hay señal débil o cuando no hay conexión, para superar esta limitante se debe contar adicionalmente con un mando manual. 


\section{REFERENCIAS}

Antúnez B., Alejandro; Mora L., David y Felmer E., Sofía. (2010). Eficiencia en Sistemas de Riego por Goteo en el Secano [en línea]. Chile: INIA, 2010 Disponible en: http://www2.inia.cl/medios/biblioteca/ta/NR36672.pdf. Consultado: 5/05/202I

Arganza R., y Arroyo, M. (2019) Posicionamiento del futuro. Un cambio de paradigma gracias a las implicaciones del "voice search" Disponible en: https://www.marketing-xxi.com/voice-search-asistentes-voz-altavocesinteligentes-seo-sem Consultado 19/06/2021

Balestrini, M. (2002). Como se elabora un proyecto de investigación. Sexta edición. Venezuela. Consultores Asociados.

Cinjordiz C. (2016). Relé electromecánico. Disponible en: https://www.infootec.net/rele-electromecanico/ Consultado I2/05/202 I

Demin Pablo (20I4).Aportes para el mejoramiento del manejo de los sistemas de riego Métodos de riego: fundamentos, usos y adaptación. Disponible en: https://inta.gob.ar/sites/default/files/inta_aportes_para_el_mejoramiento_del_ma nejo_de_los_sistemas_de_riego.pdf Consultado 21/05/2021

Hernández R,, Fernández C. y Baptista P. (20l4).Metodología de la investigación. Sexta edición. México. McGraw-Hill Interamericana.

Peláez C. Betzari (s/f) Impacto de la tecnología aplicada en la agricultura. Disponible en: http://www.sofoscorp.com/impacto-tecnologia-aplicada-agricultura/ Consultado |2/06/202|

Pineda J. (s/f). Tipos de sistema de riegos. Disponible en: https://encolombia.com/economia/agroindustria/agronomia/tipos-de-sistemas-deriego/ Consultado 12/04/2021 